TAIWANESE JOURNAL OF MATHEMATICS

Vol. 10, No. 6, pp. 1443-1464, December 2006

This paper is available online at http://www.math.nthu.edu.tw/tjm/

\title{
BOUNDEDNESS OF COMMUTATORS WITH LIPSCHITZ FUNCTIONS IN NON-HOMOGENEOUS SPACES
}

\author{
Yan Meng and Dachun Yang
}

\begin{abstract}
Under the assumption that $\mu$ is a non-doubling measure on $\mathbb{R}^{d}$, the authors obtain the boundedness of commutators generated by CalderonZygmund operators or fractional integrals with Lipschitz functions in the Lebesgue space and the Hardy space.
\end{abstract}

\section{INTRODUCTION}

During recent years, considerable attention has been paid to the study for boundedness of singular integrals with non-doubling measure; see [1, 4, 11-20, 7]. A Radon measure $\mu$ on $\mathbb{R}^{d}$ is called a non-doubling measure if it only satisfies the following growth condition that

$$
\mu(B(x, r)) \leq C r^{n}
$$

for all $x \in \mathbb{R}^{d}$ and $r>0$, where $C>0$ is a constant independent of $x$ and $r$, and $n$ is a fixed number satisfying $0<n \leq d$. The Euclidean space $\mathbb{R}^{d}$ with non-doubling measure $\mu$ is called a non-homogeneous space. Here $\mu$ is not assumed to satisfy the doubling condition. We recall that $\mu$ is said to satisfy the doubling condition if there exists some positive constant $C$ such that $\mu(B(x, 2 r)) \leq C \mu(B(x, r))$ for all $x \in \operatorname{supp}(\mu)$ and $r>0$. It is well-known that the doubling condition is a key assumption in the analysis on spaces of homogeneous type. However, some recent research has revealed that in some theories, for example, the theory of Calderon-Zygmund operators, the doubling condition is superfluous. The motivation for developing the analysis on non-homogeneous spaces and some examples of nondoubling measures can be found in [22]. We only point out that the analysis on

Received January 21, 2005; accepted January 27, 2005.

Communicated by Der-Chen Chang.

2000 Mathematics Subject Classification: Primary 47B47; Secondary 43A99.

Key words and phrases: Commutator, Calderon-Zygmund operator, Fractional integral, Lipschitz space, Lebesgue space, Hardy space. 
non-homogeneous spaces played an essential role in solving the famous Painlevé's problem by Tolsa in [21].

Let $K$ be a function on $\mathbb{R}^{d} \times \mathbb{R}^{d} \backslash\{(x, y): x=y\}$ and satisfy that

$$
|K(x, y)| \leq C|x-y|^{-n}
$$

for $x \neq y$, and if $|x-y| \geq 2\left|x-x^{\prime}\right|$,

$$
\left|K(x, y)-K\left(x^{\prime}, y\right)\right|+\left|K(y, x)-K\left(y, x^{\prime}\right)\right| \leq C \frac{\left|x-x^{\prime}\right|^{\delta}}{|x-y|^{n+\delta}}
$$

where $\delta \in(0,1]$ and $C>0$ is a positive constant. The Calderon-Zygmund operator associated to the above kernel $K$ and the measure $\mu$ is formally defined by

$$
T f(x)=\int_{\mathbb{R}^{d}} K(x, y) f(y) d \mu(y) .
$$

This integral may be not convergent for many functions. Thus we consider the truncated operator $T_{\varepsilon}$ for $\varepsilon>0$ defined by

$$
T_{\varepsilon}(f)(x)=\int_{|x-y|>\varepsilon} K(x, y) f(y) d \mu(y) .
$$

We say that $T$ is bounded on $L^{p}(\mu)$ if the operators $T_{\varepsilon}$ are bounded on $L^{p}(\mu)$ uniformly on $\varepsilon>0$. In what follows, we always assume that $T$ in (1.3) is bounded on $L^{2}(\mu)$.

Now we define multilinear commutators generated by Calderon-Zygmund operators and Lipschitz functions. First we recall the following definition of Lipschitz functions in [2].

Definition 1.1. Let $\beta>0$ and $b$ be a $\mu$-locally integrable function on $\mathbb{R}^{d}$. We say $b$ belongs to the space $\operatorname{Lip}(\beta, \mu)$ if there is a constant $C>0$ such that

$$
|b(x)-b(y)| \leq C|x-y|^{\beta}
$$

for $\mu$-almost every $x$ and $y$ in the support of $\mu$. The minimal constant $C$ appeared in (1.4) is the $\operatorname{Lip}(\beta, \mu)$ norm of $b$ and is denoted simply by $\|b\|_{\operatorname{Lip}(\beta)}$.

Let $T$ be the Calderon-Zygmund operator as in (1.3), $m \in \mathbb{N}$ and $b_{i} \in \operatorname{Lip}\left(\beta_{i}, \mu\right)$, $i=1,2, \cdots m$, the multilinear commutator $T_{\vec{b}}$ is formally defined by

$$
T_{\vec{b}}(f)(x)=\left[b_{m}, \cdots,\left[b_{2},\left[b_{1}, T\right]\right] \cdots\right](f)(x),
$$

where $\vec{b}=\left(b_{1}, b_{2}, \cdots, b_{m}\right)$, and

$$
\left[b_{1}, T\right](f)(x)=b_{1}(x) T(f)(x)-T\left(b_{1} f\right)(x) .
$$


Here in (1.5) and (1.6), $T$ stands for a weak limit as $\varepsilon \rightarrow 0$ of some subsequence of uniformly bounded operators $T_{\varepsilon}$; see [17, p. 141]. In what follows, if $m=1$ and $\vec{b}=b$, we denote $T_{\vec{b}}(f)$ simply by $T_{b}(f)$. In this paper, we will study behaviors of the commutator defined by (1.6) and the multilinear commutator defined by (1.5) in the Lebesgue space and the Hardy space. The boundedness of commutators with $B M O$ functions in some spaces of homogeneous type can be found in [8] and [9].

In Section 2, we focus on the boundedness in Lebesgue spaces. In [17], Tolsa first introduced the space $R B M O(\mu)$ and obtained the $L^{p}(\mu)$-boundedness of commutators generated by Calderon-Zygmund operators and $R B M O(\mu)$ functions for $1<p<\infty$. In [6], Hu and the authors obtained the $\left(L^{p}(\mu), L^{p}(\mu)\right)$-type estimate $(1<p<\infty)$ and the weak type endpoint estimate for multilinear commutators generated by Calderon-Zygmund operators and $R B M O(\mu)$ functions. Let $0<\beta_{i} \leq 1$ for $i=1, \cdots, m$. In this paper, we will establish the $\left(L^{p}(\mu), L^{q}(\mu)\right)$-type estimate for multilinear operators defined by (1.5) with $1<p<n /\left(\sum_{i=1}^{m} \beta_{i}\right)$ and $1 / q=1 / p-\left(\sum_{i=1}^{m} \beta_{i}\right) / n$, and their weak $\left(L^{1}(\mu), L^{n /\left(n-\sum_{i=1}^{m} \beta_{i}\right)}(\mu)\right)$-type estimate, where $0<\sum_{i=1}^{m} \beta_{i}<n$. When $m=1$, we also consider the boundedness of $T_{b}$ in the case that $n / \beta<p<\infty$ and the endpoint cases, namely, $p=n / \beta$ or $p=\infty$.

It is well-known that when $b \in B M O\left(\mathbb{R}^{d}\right)$, the commutator $T_{b}$ is not bounded on the classical Hardy space $H^{1}\left(\mathbb{R}^{d}\right)$ with the $d$-dimensional Lebesgue measure in general; see [5]. However, it is not the case for $b \in \operatorname{Lip}_{\beta}\left(\mathbb{R}^{d}\right)$; see [10]. Similar to the result in [10], we will prove that the multilinear commutator defined by (1.5) is bounded from the Hardy space $H^{1}(\mu)$ to some Lebesgue space with non-doubling measures in Section 3.

In Section 4, we also obtain corresponding results of commutators generated by fractional integrals with Lipschitz functions in the Lebesgue space and the Hardy space. We recall that for $0<\alpha<n$ and all $x \in \operatorname{supp}(\mu)$, the fractional integral $I_{\alpha}$ is defined by

$$
I_{\alpha}(f)(x)=\int_{\mathbb{R}^{d}} \frac{1}{|x-y|^{n-\alpha}} f(y) d \mu(y) .
$$

The behavior of such fractional integrals on a metric space was recently studied by García-Cuerva and Gatto in [3].

Let $0<\alpha<n$. For any $m \in \mathbb{N}, b_{i} \in \operatorname{Lip}\left(\beta_{i}, \mu\right), i=1,2, \cdots, m$, where $0<\beta_{i} \leq 1$ and $0<\alpha+\sum_{i=1}^{m} \beta_{i}<n$, the multilinear commutator, $I_{\alpha ; \vec{b}}$, is defined by $\left[b_{m}, \cdots,\left[b_{2},\left[b_{1}, I_{\alpha}\right]\right] \cdots\right]$, that is,

$$
I_{\alpha ; \vec{b}}(f)(x)=\int_{\mathbb{R}^{d}} \prod_{j=1}^{m}\left[b_{j}(x)-b_{j}(y)\right] \frac{f(y)}{|x-y|^{n-\alpha}} d \mu(y),
$$


and

$$
\left[b_{1}, I_{\alpha}\right] f(x)=\int_{\mathbb{R}^{d}}\left[b_{1}(x)-b_{1}(y)\right] \frac{f(y)}{|x-y|^{n-\alpha}} d \mu(y) .
$$

If $m=1$ and $\vec{b}=b$, we denote $I_{\alpha ; \vec{b}}$ simply by $I_{\alpha ; b}$.

In what follows, $C>0$ always denotes a constant that is independent of main parameters involved but whose value may differ from line to line. For any index $p \in[1, \infty]$, we denote by $p^{\prime}$ its conjugate index, namely, $1 / p+1 / p^{\prime}=1$.

\section{Boundedness In Lebesgue Spaces}

This section is devoted to the behavior of commutators in Lebesgue spaces.

Noting that $b_{i} \in \operatorname{Lip}\left(\beta_{i}, \mu\right), 0<\beta_{i} \leq 1$ for $1 \leq i \leq m$, we easily deduce that if $0<\sum_{i=1}^{m} \beta_{i}<n$, then for $\mu-$ a.e. $x \in \operatorname{supp}(\mu)$,

$$
\left|T_{\vec{b}}(f)(x)\right| \leq C \prod_{i=1}^{m}\left\|b_{i}\right\|_{L i p\left(\beta_{i}\right)} I_{\beta}(|f|)(x)
$$

where $\beta=\sum_{i=1}^{m} \beta_{i}$. From this and the fact that $I_{\beta}$ is bounded from $L^{p}(\mu)$ to $L^{q}(\mu)$ provided that $1<p<n / \beta$ and $1 / q=1 / p-\beta / n$ and satisfies the weak $\left(L^{1}(\mu), L^{n /(n-\beta)}(\mu)\right)$-type inequality (see [3]), it is easy to deduce the following result, which is useful in the sequel.

Theorem 2.1. Let $m \in \mathbb{N}$ and for $i=1,2, \cdots, m, b_{i} \in \operatorname{Lip}\left(\beta_{i}, \mu\right)$ with $0<\beta_{i} \leq 1$. Let $T_{\vec{b}}$ be as in (1.5). Suppose that $0<\sum_{i=1}^{m} \beta_{i}<n$, then there exists a constant $C>0$ such that

(i) for all bounded functions $f$ with compact support,

$$
\left\|T_{\vec{b}}(f)\right\|_{L^{q}(\mu)} \leq C\left\|b_{1}\right\|_{L i p\left(\beta_{1}\right)} \cdots\left\|b_{m}\right\|_{L i p\left(\beta_{m}\right)}\|f\|_{L^{p}(\mu)},
$$

where $1<p<n /\left(\sum_{i=1}^{m} \beta_{i}\right)$ and $1 / q=1 / p-\left(\sum_{i=1}^{m} \beta_{i}\right) / n$.

(ii) for all bounded functions $f$ with compact support and all $\lambda>0$,

$$
\mu\left(\left\{x \in \mathbb{R}^{d}:\left|T_{\vec{b}}(f)(x)\right|>\lambda\right\}\right) \leq C \prod_{i=1}^{m}\left\|b_{i}\right\|_{L i p\left(\beta_{i}\right)}\left(\lambda^{-1}\|f\|_{L^{1}(\mu)}\right)^{n /\left(n-\sum_{i=1}^{m} \beta_{i}\right)} .
$$

By contrast with the endpoint estimate for multilinear commutators generated by singular integrals and $R B M O(\mu)$ functions (see Theorem 2 in [6]), we can see the behavior of multilinear commutators with Lipschitz functions is quite different 
from that of multilinear commutators with $R B M O(\mu)$ functions; see also [10] for the doubling measure case.

Now we assume $m=1$. In the following, using Theorem 2.1 , we consider the boundedness of commutators defined by (1.6) for $n / \beta<p<\infty, p=n / \beta$ and $p=\infty$, respectively.

Theorem 2.2. Let $b \in \operatorname{Lip}(\beta, \mu)$ for $0<\beta \leq \delta$ and $T_{b}$ be defined as in (1.6), where $\delta$ is the same as in (1.2). If $n / \beta<p<\infty$, then there exists a constant $C>0$ such that for all bounded functions $f$ with compact support,

$$
\left\|T_{b}(f)\right\|_{L i p(\beta-n / p)} \leq C\|b\|_{L i p(\beta)}\|f\|_{L^{p}(\mu)} .
$$

Remark 2.1. The method used in the proof of Theorem 2.2 is not applicable to multilinear commutators defined by (1.5) for $m \geq 2$.

To prove Theorem 2.2, we begin with recalling some necessary notation. By a cube $Q \subset \mathbb{R}^{d}$ we mean a closed cube whose sides parallel to the axes and we denote its side length by $l(Q)$. For any $\alpha>0$ and any cube $Q, \alpha Q$ denotes the cube concentric with $Q$ and having side length $\alpha l(Q)$. For two cubes $Q_{1} \subset Q_{2}$, set

$$
K_{Q_{1}, Q_{2}}=1+\sum_{k=1}^{N_{Q_{1}, Q_{2}}} \frac{\mu\left(2^{k} Q_{1}\right)}{l\left(2^{k} Q_{1}\right)^{n}},
$$

where $N_{Q_{1}, Q_{2}}$ is the first positive integer $k$ such that $l\left(2^{k} Q_{1}\right) \geq l\left(Q_{2}\right)$.

The following characterization of the space $\operatorname{Lip}(\beta, \mu)$ for $0<\beta \leq 1$ in [2] plays a key role in the proof of Theorem 2.2.

Lemma 2.1. For a function $b \in L_{\text {loc }}^{1}(\mu)$, conditions I, II and III below are equivalent.

(i) There is a constant $C_{1} \geq 0$ such that

$$
|b(x)-b(y)| \leq C_{1}|x-y|^{\beta}
$$

for $\mu$-almost every $x$ and $y$ in the support of $\mu$.

(ii) There exist some constant $C_{2} \geq 0$ and a collection of numbers $b_{Q}$, one for each cube $Q$, such that these two properties hold: For any cube $Q$

$$
\frac{1}{\mu(2 Q)} \int_{Q}\left|b(x)-b_{Q}\right| d \mu(x) \leq C_{2} l(Q)^{\beta},
$$

and for any cube $R$ such that $Q \subset R$ and $l(R) \leq 2 l(Q)$,

$$
\left|b_{Q}-b_{R}\right| \leq C_{2} l(Q)^{\beta} \text {. }
$$


(iii) For any given $p, 1 \leq p \leq \infty$, there is a constant $C(p) \geq 0$, such that for every cube $Q$, we have

$$
\left[\frac{1}{\mu(Q)} \int_{Q}\left|b(x)-m_{Q}(b)\right|^{p} d \mu(x)\right]^{1 / p} \leq C(p) l(Q)^{\beta},
$$

where and in the sequel,

$$
m_{Q}(b)=\frac{1}{\mu(Q)} \int_{Q} b(y) d \mu(y)
$$

and also for any cube $R$ such that $Q \subset R$ and $l(R) \leq 2 l(Q)$,

$$
\left|m_{Q}(b)-m_{R}(b)\right| \leq C(p) l(Q)^{\beta} .
$$

In addition, the quantities: $\inf \left\{C_{1}\right\}, \inf \left\{C_{2}\right\}$ and $\inf \{C(p)\}$ with a fixed $p$ are equivalent.

We remark that Lemma 2.1 is a slight variant of Theorem 2.3 in [2]. To be precise, if we replace all balls in Theorem 2.3 of [2] by cubes, we then obtain Lemma 2.1 .

Remark 2.2. For $0<\beta \leq 1$, the estimate (2.2) is equivalent to

$$
\left|b_{Q}-b_{R}\right| \leq C_{2}^{\prime} K_{Q, R} l(R)^{\beta}
$$

for any two cubes $Q \subset R$; see Remark 2.7 in [2]. Note that (2.1) and (2.3) also make sense for $\beta=0$ and the space defined by using them is just the space $\operatorname{RBMO}(\mu)$ of Tolsa; see [17]. Therefore, the space $\operatorname{Lip}(\beta, \mu)$ for $0<\beta \leq 1$ can be seen as a member of a family containing $R B M O(\mu)$.

Proof of Theorem 2.2. Without loss of generality, we may assume $\|b\|_{\operatorname{Lip}(\beta)}=$ 1 . For any cube $Q$ in $\mathbb{R}^{d}$ and any cube $R$ such that $Q \subset R$ satisfying $l(R) \leq 2 l(Q)$, let

$$
a_{Q}=m_{Q}\left[T_{b}\left(f \chi_{\mathbb{R}^{d} \backslash \frac{3}{2} Q}\right)\right]
$$

and

$$
a_{R}=m_{R}\left[T_{b}\left(f \chi_{\mathbb{R}^{d} \backslash \frac{3}{2} R}\right)\right] .
$$

From Theorem 2.1, it is easy to see that $a_{Q}$ and $a_{R}$ are real numbers. By Lemma 2.1, we need to show that there exists a constant $C>0$ such that

$$
\frac{1}{\mu(2 Q)} \int_{Q}\left|T_{b}(f)(x)-a_{Q}\right| d \mu(x) \leq C\|f\|_{L^{p}(\mu)} l(Q)^{\beta-n / p}
$$


and

$$
\left|a_{Q}-a_{R}\right| \leq C\|f\|_{L^{p}(\mu)} l(Q)^{\beta-n / p} .
$$

Let us first establish the estimate (2.4). Decompose $f=f_{1}+f_{2}$, where $f_{1}=$ $f \chi_{\frac{3}{2} Q}$ and $f_{2}=f-f_{1}$. Write

$$
\begin{aligned}
& \frac{1}{\mu(2 Q)} \int_{Q}\left|T_{b}(f)(x)-a_{Q}\right| d \mu(x) \\
& \quad \leq \frac{1}{\mu(2 Q)} \int_{Q}\left|T_{b}\left(f_{1}\right)(x)\right| d \mu(x)+\frac{1}{\mu(2 Q)} \int_{Q}\left|T_{b}\left(f_{2}\right)(x)-a_{Q}\right| d \mu(x) \\
& \quad=\mathrm{I}_{1}+\mathrm{I}_{2} .
\end{aligned}
$$

Choose $1<p_{1}<n / \beta<p$ and $q_{1}$ such that $1 / q_{1}=1 / p_{1}-\beta / n$. From the Hölder inequality and Theorem 2.1, it follows that

$$
\begin{aligned}
\mathrm{I}_{1} & \leq \frac{1}{\mu(2 Q)}\left[\int_{Q}\left|T_{b}\left(f_{1}\right)(x)\right|^{q_{1}} d \mu(x)\right]^{1 / q_{1}} \mu(Q)^{1-1 / q_{1}} \\
& \leq C \frac{1}{\mu(2 Q)}\left[\int_{\frac{3}{2} Q}|f(x)|^{p_{1}} d \mu(x)\right]^{1 / p_{1}} \mu(Q)^{1-1 / q_{1}} \\
& \leq C \frac{1}{\mu(2 Q)}\left[\int_{\frac{3}{2} Q}|f(x)|^{p} d \mu(x)\right]^{1 / p} \mu\left(\frac{3}{2} Q\right)^{1 / p_{1}-1 / p} \mu(Q)^{1-1 / q_{1}} \\
& \leq C\|f\|_{L^{p}(\mu)} l(Q)^{\beta-n / p} .
\end{aligned}
$$

To estimate $\mathrm{I}_{2}$, we need to calculate the difference $\left|T_{b}\left(f_{2}\right)(x)-a_{Q}\right|$. For $\mu-a$.e. $x, y \in Q$, by (1.1), (1.2) and the Hölder inequality, we obtain

$$
\begin{aligned}
\left|T_{b}\left(f_{2}\right)(x)-T_{b}\left(f_{2}\right)(y)\right| \leq & \int_{\mathbb{R}^{d} \backslash \frac{3}{2} Q} \mid[b(x)-b(z)] K(x, z) \\
& -[b(y)-b(z)] K(y, z)|| f(z) \mid d \mu(z) \\
\leq & \int_{\mathbb{R}^{d} \backslash \frac{3}{2} Q}|[b(x)-b(z)][K(x, z)-K(y, z)]||f(z)| d \mu(z) \\
& +\int_{\mathbb{R}^{d} \backslash \frac{3}{2} Q}|[b(x)-b(z)]-[b(y)-b(z)]||K(y, z) \| f(z)| d \mu(z) \\
\leq & C \sum_{k=1}^{\infty} \int_{2^{k} \frac{3}{2} Q \backslash 2^{k-1} \frac{3}{2} Q}|x-z|^{\beta} \frac{|x-y|^{\delta}}{|x-z|^{n+\delta}}|f(z)| d \mu(z) \\
& +C \sum_{k=1}^{\infty} \int_{2^{k} \frac{3}{2} Q \backslash 2^{k-1} \frac{3}{2} Q} \frac{|x-y|^{\beta}|f(z)| d \mu(z)}{|y-z|^{n}} \mid f(z)
\end{aligned}
$$




$$
\begin{aligned}
\leq & C \sum_{k=1}^{\infty} l\left(2^{k} Q\right)^{\beta-n-\delta} l(Q)^{\delta} \int_{2^{k} \frac{3}{2} Q \backslash 2^{k-1} \frac{3}{2} Q}|f(z)| d \mu(z) \\
& +C \sum_{k=1}^{\infty} l\left(2^{k} Q\right)^{-n} l(Q)^{\beta} \int_{2^{k} \frac{3}{2} Q \backslash 2^{k-1} \frac{3}{2} Q}|f(z)| d \mu(z) \\
\leq & C\|f\|_{L^{p}(\mu)} \sum_{k=1}^{\infty} l\left(2^{k} Q\right)^{\beta-n-\delta} l(Q)^{\delta} \mu\left(2^{k} \frac{3}{2} Q\right)^{1-1 / p} \\
& +C\|f\|_{L^{p}(\mu)} \sum_{k=1}^{\infty} l\left(2^{k} Q\right)^{-n} l(Q)^{\beta} \mu\left(2^{k} \frac{3}{2} Q\right)^{1-1 / p} \\
\leq & C\|f\|_{L^{p}(\mu)} l(Q)^{\beta-n / p}\left\{\sum_{k=1}^{\infty} 2^{k(\beta-\delta-n / p)}+\sum_{k=1}^{\infty} 2^{-k n / p}\right\} \\
\leq & C\|f\|_{L^{p}(\mu)} l(Q)^{\beta-n / p},
\end{aligned}
$$

where we have used the facts that $b \in \operatorname{Lip}(\beta, \mu)$ and $\beta \leq \delta$, and that if $x, y \in Q$ and $z \in \mathbb{R}^{d} \backslash \frac{3}{2} Q$, then

$$
|K(x, z)-K(y, z)| \leq C \frac{|x-y|^{\delta}}{|x-z|^{n+\delta}},
$$

which is true by (1.2) and (1.1). From the above estimate and the choice of $a_{Q}$, we deduce that for $\mu-$ a.e. $x \in Q$,

$$
\left|T_{b}\left(f_{2}\right)(x)-a_{Q}\right| \leq C\|f\|_{L^{p}(\mu)} l(Q)^{\beta-n / p},
$$

which in turn gives us that

$$
\mathrm{I}_{2} \leq C\|f\|_{L^{p}(\mu)} l(Q)^{\beta-n / p} .
$$

Combining the estimates for $\mathrm{I}_{1}$ and $\mathrm{I}_{2}$ yields the estimate (2.4).

Now we turn to estimate (2.5). For $\mu-a$. e. $x \in Q$ and $\mu-a$. e. $y \in R$, write

$$
\begin{aligned}
& \left|T_{b}\left(f \chi_{\mathbb{R}^{d} \backslash \frac{3}{2} Q}\right)(x)-T_{b}\left(f \chi_{\mathbb{R}^{d} \backslash \frac{3}{2} R}\right)(y)\right| \\
& =\mid \int_{\mathbb{R}^{d} \backslash \frac{3}{2} R}[b(x)-b(z)] K(x, z) f(z) d \mu(z) \\
& \quad+\int_{\frac{3}{2} R \backslash \frac{3}{2} Q}[b(x)-b(z)] K(x, z) f(z) d \mu(z) \\
& \quad-\int_{\mathbb{R}^{d} \backslash \frac{3}{2} R}[b(y)-b(z)] K(y, z) f(z) d \mu(z) \mid
\end{aligned}
$$




$$
\begin{aligned}
\leq & \int_{\mathbb{R}^{d} \backslash \frac{3}{2} R}|[b(x)-b(z)] K(x, z)-[b(y)-b(z)] K(y, z)||f(z)| d \mu(z) \\
& +C \int_{\frac{3}{2} R \backslash \frac{3}{2} Q} \frac{|b(x)-b(z)|}{|x-z|^{n}}|f(z)| d \mu(z) \\
= & \mathrm{II}_{1}+\mathrm{II}_{2} .
\end{aligned}
$$

An argument similar to the estimate for $\mathrm{I}_{2}$ tells us that

$$
\mathrm{II}_{1} \leq C\|f\|_{L^{p}(\mu)} l(R)^{\beta-n / p} \leq C\|f\|_{L^{p}(\mu)} l(Q)^{\beta-n / p} .
$$

Noting that $Q \subset R$ and $l(R) \leq 2 l(Q)$, we easily obtain

$$
\begin{aligned}
\mathrm{II}_{2} & \leq C \int_{\frac{3}{2} R \backslash \frac{3}{2} Q} \frac{|f(z)|}{|x-z|^{n-\beta}} d \mu(z) \\
& \leq C\|f\|_{L^{p}(\mu)} l(Q)^{\beta-n} \mu\left(\frac{3}{2} R\right)^{1-1 / p} \\
& \leq C\|f\|_{L^{p}(\mu)} l(Q)^{\beta-n / p} .
\end{aligned}
$$

The estimates for $\mathrm{II}_{1}$ and $\mathrm{II}_{2}$ indicate that

$$
\left|a_{Q}-a_{R}\right| \leq C\|f\|_{L^{p}(\mu)} l(Q)^{\beta-n / p} .
$$

Thus, we have proved (2.5) and completed the proof of Theorem 2.2.

For the endpoint case that $p=n / \beta$, we have the following result.

Theorem 2.3. Let $b \in \operatorname{Lip}(\beta, \mu)$ for $0<\beta \leq 1$ and $T_{b}$ be defined as in (1.6). Then there is a constant $C>0$ such that for all bounded functions $f$ with compact support,

$$
\left\|T_{b}(f)\right\|_{R B M O(\mu)} \leq C\|b\|_{L i p(\beta)}\|f\|_{L^{n / \beta}(\mu)} .
$$

Here, we will not give the details for the proof of Theorem 2.3 since we can prove Theorem 2.3 by a way similar to that of Theorem 2.2. Moreover, this theorem can be deduced from Theorem 3.1 below in Section 3 by a dual argument.

For another endpoint case that $p=\infty$, using Theorem 2.2, we obtain the following result and we point out that some idea of its proof comes from [5].

Theorem 2.4. Let $b \in \operatorname{Lip}(\beta, \mu)$ for $0<\beta<\delta$ and $T_{b}$ be defined as in (1.6), where $\delta$ is the same as in (1.2). Then the following statements are equivalent.

(1) There exists a constant $C>0$ such that for all bounded functions $f$ with compact support,

$$
\left\|T_{b}(f)\right\|_{L i p(\beta)} \leq C\|b\|_{L i p(\beta)}\|f\|_{L^{\infty}(\mu)} .
$$


(2) There exists a constant $C>0$ such that the function $b$ satisfies the following conditions: For any cube $Q$ and $u \in Q$,

$$
\begin{aligned}
& \frac{1}{\mu(Q)} \int_{Q}\left|b(x)-m_{Q}(b)\right| d \mu(x)\left|\int_{\mathbb{R}^{d} \backslash 2 Q} K(u, y) f(y) d \mu(y)\right| \\
& \quad \leq C\|f\|_{L^{\infty}(\mu)} l(Q)^{\beta}
\end{aligned}
$$

and for any cube $R$ such that $Q \subset R$ and $l(R) \leq 2 l(Q)$, and any $v \in R$,

$$
\left|m_{R}(b)-m_{Q}(b)\right|\left|\int_{\mathbb{R}^{d} \backslash 2 R} K(v, y) f(y) d \mu(y)\right| \leq C\|f\|_{L^{\infty}(\mu)} l(Q)^{\beta} .
$$

Proof. For any bounded function $f$ with compact support and any cube $Q$, by Lemma 2.1 (III) with $p=1$, we need to show that

$$
\frac{1}{\mu(Q)} \int_{Q}\left|T_{b}(f)(x)-m_{Q}\left[T_{b}(f)\right]\right| d \mu(x) \leq C\|f\|_{L^{\infty}(\mu)} l(Q)^{\beta}
$$

is equivalent to (2.7), and for any cube $R$ such that $Q \subset R$ and $l(R) \leq 2 l(Q)$,

$$
\left|m_{R}\left[T_{b}(f)\right]-m_{Q}\left[T_{b}(f)\right]\right| \leq C\|f\|_{L^{\infty}(\mu)} l(Q)^{\beta}
$$

is equivalent to $(2.8)$.

First we prove that (2.9) is equivalent to (2.7). For $x \in Q$, write

$$
\begin{aligned}
T_{b}(f)(x)-m_{Q}\left[T_{b}(f)\right] \\
=T_{b}\left(f \chi_{2 Q}\right)(x)-\frac{1}{\mu(Q)} \int_{Q} T_{b}\left(f \chi_{2 Q}\right)(z) d \mu(z) \\
\quad+T_{b}\left(f \chi_{\mathbb{R}^{d} \backslash 2 Q}\right)(x)-\frac{1}{\mu(Q)} \int_{Q} T_{b}\left(f \chi_{\mathbb{R}^{d} \backslash 2 Q}\right)(z) d \mu(z) \\
=T_{b}\left(f \chi_{2 Q}\right)(x)-m_{Q}\left[T_{b}\left(f \chi_{2 Q}\right)\right]+\left[b(x)-m_{Q}(b)\right] T\left(f \chi_{\mathbb{R}^{d} \backslash 2 Q}\right)(x) \\
\quad-T\left(\left[b-m_{Q}(b)\right] f \chi_{\mathbb{R}^{d} \backslash 2 Q}\right)(x)-\frac{1}{\mu(Q)} \int_{Q}\left[b(z)-m_{Q}(b)\right] T\left(f \chi_{\mathbb{R}^{d} \backslash 2 Q}\right)(z) d \mu(z) \\
\quad+\frac{1}{\mu(Q)} \int_{Q} T\left(\left[b-m_{Q}(b)\right] f \chi_{\mathbb{R}^{d} \backslash 2 Q}\right)(z) d \mu(z) .
\end{aligned}
$$

From this, it follows that for any $x, u \in Q$, 


$$
\begin{aligned}
T_{b}(f) & (x)-m_{Q}\left[T_{b}(f)\right] \\
= & T_{b}\left(f \chi_{2 Q}\right)(x)-m_{Q}\left[T_{b}\left(f \chi_{2 Q}\right)\right] \\
& +\left[b(x)-m_{Q}(b)\right]\left[T\left(f \chi_{\mathbb{R}^{d} \backslash 2 Q}\right)(x)-T\left(f \chi_{\mathbb{R}^{d} \backslash 2 Q}\right)(u)\right] \\
& +\left[b(x)-m_{Q}(b)\right] T\left(f \chi_{\mathbb{R}^{d} \backslash 2 Q}\right)(u) \\
& -\frac{1}{\mu(Q)} \int_{Q}\left[b(z)-m_{Q}(b)\right]\left[T\left(f \chi_{\mathbb{R}^{d} \backslash 2 Q}\right)(z)-T\left(f \chi_{\mathbb{R}^{d} \backslash 2 Q}\right)(u)\right] d \mu(z) \\
& +\frac{1}{\mu(Q)} \int_{Q}\left[T\left(\left[b-m_{Q}(b)\right] f \chi_{\mathbb{R}^{d} \backslash 2 Q}\right)(z)-T\left(\left[b-m_{Q}(b)\right] f \chi_{\mathbb{R}^{d} \backslash 2 Q}\right)(x)\right] d \mu(z) .
\end{aligned}
$$

Now, if we define

$$
\begin{aligned}
& \eta_{1}(x)=T_{b}\left(f \chi_{2 Q}\right)(x), \\
& \eta_{2}(x, u)=\left[b(x)-m_{Q}(b)\right]\left[T\left(f \chi_{\mathbb{R}^{d} \backslash 2 Q}\right)(x)-T\left(f \chi_{\mathbb{R}^{d} \backslash 2 Q}\right)(u)\right], \\
& \eta_{3}(x)=\frac{1}{\mu(Q)} \int_{Q}\left[T\left(\left[b-m_{Q}(b)\right] f \chi_{\mathbb{R}^{d} \backslash 2 Q}\right)(z)-T\left(\left[b-m_{Q}(b)\right] f \chi_{\mathbb{R}^{d} \backslash 2 Q}\right)(x)\right] d \mu(z)
\end{aligned}
$$

and

$$
\eta_{4}(x, u)=\left[b(x)-m_{Q}(b)\right] T\left(f \chi_{\mathbb{R}^{d} \backslash 2 Q}\right)(u),
$$

then we easily see

$$
\begin{aligned}
T_{b}(f)(x)-m_{Q}\left[T_{b}(f)\right]= & \eta_{1}(x)-m_{Q}\left(\eta_{1}\right)+\eta_{2}(x, u) \\
& -m_{Q}\left[\eta_{2}(\cdot, u)\right]+\eta_{3}(x)+\eta_{4}(x, u) .
\end{aligned}
$$

We claim that

$$
\begin{gathered}
\frac{1}{\mu(Q)} \int_{Q}\left|\eta_{1}(x)-m_{Q}\left(\eta_{1}\right)\right| d \mu(x) \leq C\|f\|_{L^{\infty}(\mu)} l(Q)^{\beta} \\
\frac{1}{\mu(Q)} \int_{Q}\left|\eta_{2}(x, u)\right| d \mu(x) \leq C\|f\|_{L^{\infty}(\mu)} l(Q)^{\beta}
\end{gathered}
$$

and

$$
\frac{1}{\mu(Q)} \int_{Q}\left|\eta_{3}(x)\right| d \mu(x) \leq C\|f\|_{L^{\infty}(\mu)} l(Q)^{\beta} .
$$


Take $n / \beta<p_{2}<\infty$. Theorem 2.2 together with the fact that $f \chi_{2 Q} \in L^{p_{2}}(\mu)$ gives us that

$\frac{1}{\mu(Q)} \int_{Q}\left|\eta_{1}(x)-m_{Q}\left(\eta_{1}\right)\right| d \mu(x) \leq C\left\|f \chi_{2 Q}\right\|_{L^{p_{2}(\mu)}} l(Q)^{\beta-n / p_{2}} \leq C\|f\|_{L^{\infty}(\mu)} l(Q)^{\beta}$.

For (2.13), noting $u \in Q$, by (2.6) and Lemma 2.1 (III) with $p=1$, we obtain

$$
\begin{aligned}
& \frac{1}{\mu(Q)} \int_{Q}\left|\eta_{2}(x, u)\right| d \mu(x) \\
& \quad \leq \frac{1}{\mu(Q)} \int_{Q}\left|b(x)-m_{Q}(b)\right| \int_{\mathbb{R}^{d} \backslash 2 Q}|K(x, z)-K(u, z)| f(z) \mid d \mu(z) d \mu(x) \\
& \quad \leq C\|f\|_{L^{\infty}(\mu)} \frac{1}{\mu(Q)} \int_{Q}\left|b(x)-m_{Q}(b)\right| \sum_{k=1}^{\infty} \int_{2^{k+1} Q \backslash 2^{k} Q} \frac{|x-u|^{\delta}}{|x-z|^{n+\delta}} d \mu(z) d \mu(x) \\
& \quad \leq C\|f\|_{L^{\infty}(\mu)} l(Q)^{\beta} .
\end{aligned}
$$

Finally we prove (2.14). For $x, z \in Q$, by (2.6),

$$
\begin{aligned}
& \left|T\left(\left[b-m_{Q}(b)\right] f \chi_{\mathbb{R}^{d} \backslash 2 Q}\right)(z)-T\left(\left[b-m_{Q}(b)\right] f \chi_{\mathbb{R}^{d} \backslash 2 Q}\right)(x)\right| \\
& \quad \leq \int_{\mathbb{R}^{d} \backslash 2 Q}|K(z, y)-K(x, y)|\left|b(y)-m_{Q}(b)\right||f(y)| d \mu(y) \\
& \quad \leq C\|f\|_{L^{\infty}(\mu)} \sum_{k=1}^{\infty} \int_{2^{k+1} Q \backslash 2^{k} Q} \frac{|x-z|^{\delta}}{|x-y|^{n+\delta}}\left|b(y)-m_{Q}(b)\right| d \mu(y) \\
& \quad \leq C\|f\|_{L^{\infty}(\mu)} \sum_{k=1}^{\infty} l(Q)^{\delta} l\left(2^{k} Q\right)^{-n-\delta} l\left(2^{k} Q\right)^{n} l\left(2^{k} Q\right)^{\beta} \\
& \quad \leq C\|f\|_{L^{\infty}(\mu)} l(Q)^{\beta},
\end{aligned}
$$

where we used the fact that $\beta<\delta$. From this, it follows that

$$
\frac{1}{\mu(Q)} \int_{Q}\left|\eta_{3}(x)\right| d \mu(x) \leq C\|f\|_{L^{\infty}(\mu)} l(Q)^{\beta} .
$$

Now the equivalence between (2.7) and (2.9) follows easily. Assume first (2.9) holds. By (2.11), for $x, u \in Q$, we have

$$
\begin{aligned}
\eta_{4}(x, u)= & \left\{T_{b}(f)(x)-m_{Q}\left[T_{b}(f)\right]\right\}-\left\{\eta_{1}(x)-m_{Q}\left(\eta_{1}\right)\right\} \\
& -\left\{\eta_{2}(x, u)-m_{Q}\left[\eta_{2}(\cdot, u)\right]\right\}-\eta_{3}(x) .
\end{aligned}
$$


Taking the mean over $Q$ in $x$ and using the boundedness of $T_{b}$ and the estimates (2.12), (2.13) and (2.14), we obtain

$$
\begin{aligned}
\frac{1}{\mu(Q)} \int_{Q}\left|\eta_{4}(x, u)\right| d \mu(x) \leq & \frac{1}{\mu(Q)} \int_{Q}\left|T_{b}(f)(x)-m_{Q}\left[T_{b}(f)\right]\right| d \mu(x) \\
& +\frac{1}{\mu(Q)} \int_{Q}\left|\eta_{1}(x)-m_{Q}\left(\eta_{1}\right)\right| d \mu(x) \\
& +\frac{1}{\mu(Q)} \int_{Q}\left|\eta_{2}(x, u)-m_{Q}\left[\eta_{2}(\cdot, u)\right]\right| d \mu(x) \\
& +\frac{1}{\mu(Q)} \int_{Q}\left|\eta_{3}(x)\right| d \mu(x) \\
\leq & C\|f\|_{L^{\infty}(\mu)} l(Q)^{\beta} .
\end{aligned}
$$

This means that for any cube $Q$ and $u \in Q$, (2.7) holds. Conversely, assume (2.7) holds. As we have just seen, this is equivalent to (2.15). Therefore going back to (2.11) and inserting the estimates (2.12), (2.13), (2.14) and (2.15), we obtain (2.9).

Now we turn our attention to verify that (2.8) is equivalent to (2.10). For any cube $R$ such that $Q \subset R$ and $l(R) \leq 2 l(Q)$, write

$$
\begin{aligned}
m_{Q}\left[T_{b}(f)\right]-m_{R}\left[T_{b}(f)\right]= & \left\{m_{Q}\left[T_{b}\left(f \chi_{2 R}\right)\right]-m_{R}\left[T_{b}\left(f \chi_{2 R}\right)\right]\right\} \\
& +\left\{m_{Q}\left[T_{b}\left(f \chi_{\mathbb{R}^{d} \backslash 2 R}\right)\right]-m_{R}\left[T_{b}\left(f \chi_{\mathbb{R}^{d} \backslash 2 R}\right)\right]\right\} \\
= & \mathrm{H}_{1}+\mathrm{H}_{2} .
\end{aligned}
$$

From Theorem 2.2 and Lemma 2.1 (III), it follows that for any $n / \beta<p<\infty$,

$$
\left|\mathrm{H}_{1}\right| \leq C\left\|f \chi_{2 R}\right\|_{L^{p}(\mu)} l(Q)^{\beta-n / p} \leq C\|f\|_{L^{\infty}(\mu)} l(Q)^{\beta} .
$$

To estimate $\mathrm{H}_{2}$, for any $x \in Q$ and $v \in R$, we write

$$
\begin{aligned}
& T_{b}\left(f \chi_{\mathbb{R}^{d} \backslash 2 R}\right)(x)-m_{R}\left(T_{b} f \chi_{\mathbb{R}^{d} \backslash 2 R}\right) \\
& \quad=\eta_{2}^{\prime}(x, v)-m_{R}\left[\eta_{2}^{\prime}(\cdot, v)\right]+\eta_{3}^{\prime}(x)+\eta_{4}^{\prime}(x, v),
\end{aligned}
$$

where

$$
\begin{aligned}
& \eta_{2}^{\prime}(x, v)=\left[b(x)-m_{R}(b)\right]\left[T\left(f \chi_{\mathbb{R}^{d} \backslash 2 R}\right)(x)-T\left(f \chi_{\mathbb{R}^{d} \backslash 2 R}\right)(v)\right] \\
& \eta_{3}^{\prime}(x)=m_{R}\left[T\left(\left[b-m_{R}(b)\right] f \chi_{\mathbb{R}^{d} \backslash 2 R}\right)\right]-T\left(\left[b-m_{R}(b)\right] f \chi_{\mathbb{R}^{d} \backslash 2 R}\right)(x)
\end{aligned}
$$

and

$$
\eta_{4}^{\prime}(x, v)=\left[b(x)-m_{R}(b)\right] T\left(f \chi_{\mathbb{R}^{d} \backslash 2 R}\right)(v) .
$$


Some computations similar to that for (2.13) and (2.14) tell us that

$$
\begin{aligned}
& m_{R}\left[\eta_{2}^{\prime}(\cdot, v)\right] \leq C\|f\|_{L^{\infty}(\mu)} l(R)^{\beta} \leq C\|f\|_{L^{\infty}(\mu)} l(Q)^{\beta}, \\
& m_{Q}\left[\eta_{2}^{\prime}(\cdot, v)\right] \leq C\|f\|_{L^{\infty}(\mu)} l(Q)^{\beta}
\end{aligned}
$$

and

$$
m_{Q}\left(\eta_{3}^{\prime}\right) \leq C\|f\|_{L^{\infty}(\mu)} l(R)^{\beta} \leq C\|f\|_{L^{\infty}(\mu)} l(Q)^{\beta} .
$$

Taking the mean over $x \in Q$ in (2.17) and by (2.16), we obtain

$$
\begin{aligned}
m_{Q}\left[\eta_{4}^{\prime}(\cdot, v)\right]= & \left\{m_{Q}\left[T_{b}(f)\right]-m_{R}\left[T_{b}(f)\right]\right\}-H_{1} \\
& -m_{Q}\left[\eta_{2}^{\prime}(\cdot, v)\right]+m_{R}\left[\eta_{2}^{\prime}(\cdot, v)\right]-m_{Q}\left(\eta_{3}^{\prime}\right) .
\end{aligned}
$$

An argument similar to the proof of the equivalence between (2.7) and (2.9) tells us that (2.8) is equivalent to (2.10). This finishes the proof of Theorem 2.4.

\section{Boundedness in Hardy Space $H^{1}(\mu)$}

To study the boundedness of multilinear commutators generated by CalderonZygmund operators with Lipschitz functions in the Hardy space $H^{1}(\mu)$ of Tolsa in $[17,19]$, we first recall the definition of the "grand" maximal operator $M_{\Phi}$ of Tolsa in [19].

Definition 3.1. Given $f \in L_{\mathrm{loc}}^{1}(\mu)$, we define

$$
M_{\Phi} f(x)=\sup _{\varphi \sim x}\left|\int_{\mathbb{R}^{d}} f \varphi d \mu\right|,
$$

where the notation $\varphi \sim x$ means that $\varphi \in L^{1}(\mu) \cap C^{1}\left(\mathbb{R}^{d}\right)$ and satisfies

(i) $\|\varphi\|_{L^{1}(\mu)} \leq 1$,

(ii) $0 \leq \varphi(y) \leq \frac{1}{|y-x|^{n}}$ for all $y \in \mathbb{R}^{d}$, and

(iii) $|\nabla \varphi(y)| \leq \frac{1}{|y-x|^{n+1}}$ for all $y \in \mathbb{R}^{d}$.

Based on Theorem 1.2 of Tolsa in [19], we can define the Hardy space $H^{1}(\mu)$ as follows; see also [17].

Definition 3.2. The Hardy space $H^{1}(\mu)$ is the set of all functions $f \in L^{1}(\mu)$ satisfying that $\int_{\mathbb{R}^{d}} f d \mu=0$ and $M_{\Phi} f \in L^{1}(\mu)$. Moreover, we define the norm of $f \in H^{1}(\mu)$ by

$$
\|f\|_{H^{1}(\mu)}=\|f\|_{L^{1}(\mu)}+\left\|M_{\Phi} f\right\|_{L^{1}(\mu)} .
$$


Using Theorem 2.1, we can obtain the following boundedness of multilinear commutators in the Hardy space $H^{1}(\mu)$.

Theorem 3.1. Let $m \in \mathbb{N}$ and for $i=1,2, \cdots, m, b_{i} \in \operatorname{Lip}\left(\beta_{i}, \mu\right)$ and $0<\beta_{i} \leq 1$. Let $T_{\vec{b}}$ be as in (1.5). Suppose that $0<\sum_{i=1}^{m} \beta_{i}<n$ and $1 / q=$ $1-\left(\sum_{i=1}^{m} \beta_{i}\right) / n$. Then $T_{\vec{b}}$ is bounded from $H^{1}(\mu)$ to $L^{q}(\mu)$ with the operator norm at most $C\left\|b_{1}\right\|_{L i p\left(\beta_{1}\right)} \cdots\left\|b_{m}\right\|_{L i p\left(\beta_{m}\right)}$.

Remark 3.1. In [17], Tolsa showed that the space $R B M O(\mu)$ is the dual of the Hardy space $H^{1}(\mu)$ as in the doubling case. Using this and the fact that $L^{n / \beta}(\mu)$ is the dual of $L^{n /(n-\beta)}(\mu)$, we can deduce Theorem 2.3 from Theorem 3.1. We omit the details.

To prove Theorem 3.1, we first recall the definition of the atomic Hardy space $H_{a t b}^{1, \infty}(\mu)$, which has been proved to be the same space as the Hardy space $H^{1}(\mu)$; see $[17,19]$.

Definition 3.3. Let $\rho>1$. A function $h \in L_{\text {loc }}^{1}(\mu)$ is called a atomic block if

(1) there exists some cube $R$ such that $\operatorname{supp}(h) \subset R$,

(2) $\int_{\mathbb{R}^{d}} h(x) d \mu(x)=0$,

(3) for $i=1,2$, there are functions $a_{i}$ supported on cubes $Q_{i} \subset R$ and numbers $\lambda_{i} \in \mathbb{R}$ such that $h=\lambda_{1} a_{1}+\lambda_{2} a_{2}$, and

$$
\left\|a_{i}\right\|_{L^{\infty}(\mu)} \leq\left[\mu\left(\rho Q_{i}\right) K_{Q_{i}, R}\right]^{-1} .
$$

Then we define

$$
|h|_{H_{\text {atb }}^{1, \infty}(\mu)}=\left|\lambda_{1}\right|+\left|\lambda_{2}\right| .
$$

We say that $f \in H_{\text {atb }}^{1, \infty}(\mu)$ if there are atomic blocks $\left\{h_{j}\right\}_{j \in \mathbb{N}}$ such that

$$
f=\sum_{j=1}^{\infty} h_{j}
$$

with $\sum_{j=1}^{\infty}\left|h_{j}\right|_{H_{a t b}^{1, \infty}(\mu)}<\infty$. The $H_{a t b}^{1, \infty}(\mu)$ norm of $f$ is defined by

$$
\|f\|_{H_{a t b}^{1, \infty}(\mu)}=\inf \left\{\sum_{j}\left|h_{j}\right|_{H_{a t b}^{1, \infty}(\mu)}\right\},
$$

where the infimum is taken over all possible decompositions of $f$ in atomic blocks. 
The definition of $H_{a t b}^{1, \infty}(\mu)$ does not depend on the constant $\rho>1$, which was proved by Tolsa in [17].

Proof of Theorem 3.1. For simplicity, set $\beta=\sum_{i=1}^{m} \beta_{i}$. Without loss of generality, we may assume that $\left\|b_{i}\right\|_{L i p\left(\beta_{i}\right)}=1$ for $i=1, \cdots, m$. It is easy to see that we only need to prove the theorem for atomic blocks $h$ as in Definition 3.3 with $\rho=4$. Let $R$ be a cube such that $\operatorname{supp}(h) \subset R, \int_{\mathbb{R}^{d}} h(x) d \mu(x)=0$, and

$$
h(x)=\lambda_{1} a_{1}(x)+\lambda_{2} a_{2}(x),
$$

where $\lambda_{i}$ for $i=1,2$ is a real number, $|h|_{H_{a t b}^{1, \infty}(\mu)}=\left|\lambda_{1}\right|+\left|\lambda_{2}\right|, a_{i}$ for $i=1,2$ is a bounded function supported on some cube $Q_{i} \subset R$ and satisfies

$$
\left\|a_{i}\right\|_{L^{\infty}(\mu)} \leq\left[\mu\left(4 Q_{i}\right) K_{Q_{i}, R}\right]^{-1} .
$$

Write

$$
\begin{aligned}
\left\|T_{\vec{b}}(h)\right\|_{L^{q}(\mu)} & \leq\left[\int_{2 R}\left|T_{\vec{b}}(h)(x)\right|^{q} d \mu(x)\right]^{1 / q}+\left[\int_{\mathbb{R}^{d} \backslash 2 R}\left|T_{\vec{b}}(h)(x)\right|^{q} d \mu(x)\right]^{1 / q} \\
& =\mathrm{L}_{1}+\mathrm{L}_{2} .
\end{aligned}
$$

By (3.1), we can further decompose

$$
\mathrm{L}_{1} \leq\left|\lambda_{1}\right|\left[\int_{2 R}\left|T_{\vec{b}}\left(a_{1}\right)(x)\right|^{q} d \mu(x)\right]^{1 / q}+\left|\lambda_{2}\right|\left[\int_{2 R}\left|T_{\vec{b}}\left(a_{2}\right)(x)\right|^{q} d \mu(x)\right]^{1 / q}=\mathrm{J}_{1}+\mathrm{J}_{2} .
$$

To estimate $\mathrm{J}_{1}$, we write

$$
\begin{aligned}
\mathrm{J}_{1} & \leq\left|\lambda_{1}\right|\left[\int_{2 Q_{1}}\left|T_{\vec{b}}\left(a_{1}\right)(x)\right|^{q} d \mu(x)\right]^{1 / q}+\left|\lambda_{1}\right|\left[\int_{2 R \backslash 2 Q_{1}}\left|T_{\vec{b}}\left(a_{1}\right)(x)\right|^{q} d \mu(x)\right]^{1 / q} \\
& =\mathrm{J}_{11}+\mathrm{J}_{12} .
\end{aligned}
$$

Choose $1<p_{3}<n / \beta$ and $q_{3}$ such that $1 / q_{3}=1 / p_{3}-\beta / n$; then $1<q<q_{3}$. The Hölder inequality, the fact that $K_{Q_{1}, R} \geq 1$, and the $\left(L^{p_{3}}(\mu), L^{q_{3}}(\mu)\right)$-type estimate satisfied by $T_{\vec{b}}$, which is indicated by Theorem 2.1 in Section 2 , tell us that

$$
\begin{aligned}
\mathrm{J}_{11} & \leq\left|\lambda_{1}\right|\left[\int_{2 Q_{1}}\left|T_{\vec{b}}\left(a_{1}\right)(x)\right|^{q_{3}} d \mu(x)\right]^{1 / q_{3}} \mu\left(2 Q_{1}\right)^{1 / q-1 / q_{3}} \\
& \leq C\left|\lambda_{1}\right|\left\|a_{1}\right\|_{L^{p_{3}(\mu)}} \mu\left(2 Q_{1}\right)^{1 / q-1 / q_{3}} \\
& \leq C\left|\lambda_{1}\right|\left\|a_{1}\right\|_{L^{\infty}(\mu)} \mu\left(2 Q_{1}\right)^{1 / p_{3}+1 / q-1 / q_{3}} \\
& \leq C\left|\lambda_{1}\right| .
\end{aligned}
$$


Denote $N_{2 Q_{1}, 2 R}$ simply by $N_{1}$. Invoking the fact that $\left\|a_{1}\right\|_{L^{\infty}(\mu)} \leq\left[\mu\left(4 Q_{1}\right)\right.$ $\left.K_{Q_{1}, R}\right]^{-1}$, we have

$$
\begin{aligned}
\mathrm{J}_{12} & \leq C\left|\lambda_{1}\right|\left\{\sum_{k=1}^{N_{1}+1} \int_{2^{k+1} Q_{1} \backslash 2^{k} Q_{1}}\left[\int_{Q_{1}} \frac{\prod_{i=1}^{m}\left|b_{i}(x)-b_{i}(y)\right|}{|x-y|^{n}}\left|a_{1}(y)\right| d \mu(y)\right]^{q} d \mu(x)\right\}^{1 / q} \\
& \leq C\left|\lambda_{1}\right|\left\{\sum_{k=1}^{N_{1}+1} l\left(2^{k} Q_{1}\right)^{q(\beta-n)} \int_{2^{k+1} Q_{1} \backslash 2^{k} Q_{1}}\left[\int_{Q_{1}}\left|a_{1}(y)\right| d \mu(y)\right]^{q} d \mu(x)\right\}^{1 / q} \\
& \leq C\left|\lambda_{1}\right|\left\{\sum_{k=1}^{N_{1}+1} l\left(2^{k} Q_{1}\right)^{q(\beta-n)} \mu\left(2^{k+1} Q_{1}\right)\left\|a_{1}\right\|_{L^{\infty}(\mu)}^{q} \mu\left(Q_{1}\right)^{q}\right\}^{1 / q} \\
& \leq C\left|\lambda_{1}\right|\left\{K_{Q_{1}, R}^{-q} \sum_{k=2}^{N_{1}+2} \frac{\mu\left(2^{k} Q_{1}\right)}{l\left(2^{k} Q_{1}\right)^{n}}\right\}^{1 / q} \\
& \leq C\left|\lambda_{1}\right|
\end{aligned}
$$

where we have used the fact that

$$
\sum_{k=2}^{N_{1}+2} \frac{\mu\left(2^{k} Q_{1}\right)}{l\left(2^{k} Q_{1}\right)^{n}} \leq C K_{Q_{1}, R}
$$

see $[17,19]$. The estimates for $J_{11}$ and $J_{12}$ give the desired estimate for $J_{1}$. An argument similar to the estimate for $\mathrm{J}_{1}$ tells us that

$$
\mathrm{J}_{2} \leq C\left|\lambda_{2}\right| \text {. }
$$

Combining the estimates for $\mathrm{J}_{1}$ and $\mathrm{J}_{2}$ yields the desired estimate for $\mathrm{L}_{1}$.

Now we turn our attention to the estimate for $\mathrm{L}_{2}$. For $1 \leq i \leq m$, we denote by $C_{i}^{m}$ the family of all finite subset $\sigma=\{\sigma(1), \cdots, \sigma(i)\}$ of $\{1,2, \cdots, m\}$ with $i$ different elements. For any $\sigma \in C_{i}^{m}$, the complementary sequence $\sigma^{\prime}$ is given by $\sigma^{\prime}=\{1,2 \cdots, m\} \backslash \sigma$. For any $\sigma=\{\sigma(1), \sigma(2), \cdots, \sigma(i)\} \in C_{i}^{m}$, set $\beta_{\sigma}=\beta_{\sigma(1)}+\cdots+\beta_{\sigma(i)}$ and $\beta_{\sigma^{\prime}}=\beta-\beta_{\sigma}$. For $1 \leq i \leq m$, all $\sigma \in C_{i}^{m}$, all $y \in \mathbb{R}^{d}$ and all cubes $R$, write

$$
\left[b(y)-m_{R}(b)\right]_{\sigma}=\left[b_{\sigma(1)}(y)-m_{R}\left(b_{\sigma(1)}\right)\right] \cdots\left[b_{\sigma(i)}(y)-m_{R}\left(b_{\sigma(i)}\right)\right] .
$$

Let $x_{R}$ be the center of $R$. With the aid of the formula

$$
\prod_{i=1}^{m}\left[b_{i}(x)-b_{i}(y)\right]=\sum_{i=0}^{m} \sum_{\sigma \in C_{i}^{m}}\left[b(x)-m_{R}(b)\right]_{\sigma}\left[m_{R}(b)-b(y)\right]_{\sigma^{\prime}}
$$

the estimate (2.6), and the fact that

$$
\int_{R} h(x) d \mu(x)=0
$$


we obtain

$L_{2}$

$$
\begin{aligned}
& \leq C\left\{\sum_{k=1}^{\infty} \int_{2^{k+1} R \backslash 2^{k} R}\left|\prod_{i=1}^{m}\left[b_{i}(x)-m_{R}\left(b_{i}\right)\right] \int_{R} K(x, y) h(y) d \mu(y)\right|^{q} d \mu(x)\right\}^{1 / q} \\
& +C\left\{\sum_{k=1}^{\infty} \int_{2^{k+1} R \backslash 2^{k} R} \mid \sum_{i=1}^{m} \sum_{\sigma \in C_{i}^{m}}\left[b(x)-m_{R}(b)\right]_{\sigma^{\prime}}\right. \\
& \left.\times\left.\int_{R}\left[m_{R}(b)-b(y)\right]_{\sigma} K(x, y) h(y) d \mu(y)\right|^{q} d \mu(x)\right\}^{1 / q} \\
& \leq C\left\{\sum_{k=1}^{\infty} \int_{2^{k+1} R \backslash 2^{k} R}\left|l\left(2^{k} R\right)^{\beta} \int_{R}\left[K(x, y)-K\left(x, x_{R}\right)\right] h(y) d \mu(y)\right|^{q} d \mu(x)\right\}^{1 / q} \\
& +C\left\{\sum_{k=1}^{\infty} \int_{2^{k+1} R \backslash 2^{k} R}\left|\sum_{i=1}^{m} \sum_{\sigma \in C_{i}^{m}} l\left(2^{k} R\right)^{\beta_{\sigma^{\prime}}} \int_{R} \frac{l(R)^{\beta_{\sigma}}}{|x-y|^{n}}\right| h(y)|d \mu(y)|^{q} d \mu(x)\right\}^{1 / q} \\
& \leq C\left\{\sum_{k=1}^{\infty} \int_{2^{k+1} R \backslash 2^{k} R}\left|l\left(2^{k} R\right)^{\beta} \int_{R} \frac{\left|y-x_{R}\right|^{\delta}}{|x-y|^{n+\delta}}\left(\sum_{i=1}^{2}\left|\lambda_{i}\right|\left|a_{i}(y)\right|\right) d \mu(y)\right|^{q} d \mu(x)\right\}^{1 / q} \\
& +C\left\{\sum_{k=1}^{\infty} \int_{2^{k+1} R \backslash 2^{k} R}\left|\sum_{i=1}^{m} \sum_{\sigma \in C_{i}^{m}} \frac{l(R)^{\beta_{\sigma}}}{l\left(2^{k} R\right)^{n-\beta_{\sigma^{\prime}}}} \int_{R}\left(\sum_{i=1}^{2}\left|\lambda_{i}\right|\left|a_{i}(y)\right|\right) d \mu(y)\right|^{q} d \mu(x)\right\}^{1 / q} \\
& \leq C\left(\sum_{i=1}^{2}\left|\lambda_{i}\right|\right)\left\{\sum_{k=1}^{\infty} l\left(2^{k} R\right)^{q(\beta-n-\delta)} l(R)^{\delta q} \mu\left(2^{k+1} R\right)\right\}^{1 / q} \\
& +C\left(\sum_{i=1}^{2}\left|\lambda_{i}\right|\right)\left\{\sum_{k=1}^{\infty} \sum_{i=1}^{m} \sum_{\sigma \in C_{i}^{m}} l\left(2^{k} R\right)^{q\left(\beta_{\sigma^{\prime}}-n\right)} l(R)^{\beta_{\sigma} q} \mu\left(2^{k+1} R\right)\right\}^{1 / q} \\
& \leq C\left(\sum_{i=1}^{2}\left|\lambda_{i}\right|\right)\left(\sum_{k=1}^{\infty} 2^{-q \delta k}\right)^{1 / q}+C\left(\sum_{i=1}^{2}\left|\lambda_{i}\right|\right)\left(\sum_{k=1}^{\infty} \sum_{i=1}^{m} \sum_{\sigma \in C_{i}^{m}} 2^{-q \beta_{\sigma} k}\right)^{1 / q} \\
& \leq C\left(\sum_{i=1}^{2}\left|\lambda_{i}\right|\right) \text {. }
\end{aligned}
$$

Combining the estimates for $\mathrm{L}_{1}$ and $\mathrm{L}_{2}$ yields that

$$
\left\|T_{\vec{b}}(h)\right\|_{L^{q}(\mu)} \leq C|h|_{H_{a t b}^{1, \infty}(\mu)}
$$

and this finishes the proof of Theorem 3.1. 


\section{Commutators of Fractional Integrals}

In this section, we study the boundedness of commutators defined by (1.8) and multilinear commutators defined by (1.7) in the Lebesgue space and the Hardy space.

From the facts that for $\mu-a . e . x \in \operatorname{supp}(\mu)$,

$$
\left|I_{\alpha ; \vec{b}}(f)(x)\right| \leq C \prod_{i=1}^{m}\left\|b_{i}\right\|_{L i p\left(\beta_{i}\right)} I_{\alpha+\beta}(|f|)(x),
$$

where $\beta=\sum_{i=1}^{m} \beta_{i}$ and $0<\alpha+\beta<n$, and the boundedness of $I_{\alpha+\beta}$ in [3], we easily deduce the following result.

Theorem 4.1. Let $0<\alpha<n, m \in \mathbb{N}$ and for $i=1,2, \cdots, m, b_{i} \in$ Lip $\left(\beta_{i}, \mu\right)$, where $0<\beta_{i} \leq 1$. Let $I_{\alpha ; \vec{b}}$ be as in (1.7). Suppose that $0<\alpha+\sum_{i=1}^{m} \beta_{i}<$ $n$, then

(i) there exists a constant $C>0$ such that for all bounded functions $f$ with compact support,

$$
\left\|I_{\alpha ; \vec{b}}(f)\right\|_{L^{q}(\mu)} \leq C\left\|b_{1}\right\|_{L i p\left(\beta_{1}\right)} \cdots\left\|b_{m}\right\|_{L i p\left(\beta_{m}\right)}\|f\|_{L^{p}(\mu)},
$$

where $1<p<n /\left(\alpha+\sum_{i=1}^{m} \beta_{i}\right)$ and $1 / q=1 / p-\left(\alpha+\sum_{i=1}^{m} \beta_{i}\right) / n$.

(ii) there exists a constant $C>0$ such that for all bounded functions $f$ with compact support and any $\lambda>0$,

$$
\begin{aligned}
& \mu\left(\left\{x \in \mathbb{R}^{d}:\left|I_{\alpha ; \vec{b}}(f)(x)\right|>\lambda\right\}\right) \\
& \quad \leq C\left\|b_{1}\right\|_{L i p\left(\beta_{1}\right)} \cdots\left\|b_{m}\right\|_{L i p\left(\beta_{m}\right)}\left(\lambda^{-1}\|f\|_{L^{1}(\mu)}\right)^{n /\left(n-\alpha-\sum_{i=1}^{m} \beta_{i}\right)} .
\end{aligned}
$$

Using Theorem 4.1, by a method similar to the proof of Theorem 3.1, we can obtain the following boundedness in the Hardy space $H^{1}(\mu)$ of fractional multilinear commutators (1.7). We omit the details.

Theorem 4.2. Let $0<\alpha<n, m \in \mathbb{N}$ and for $i=1,2, \cdots, m, b_{i} \in$ $\operatorname{Lip}\left(\beta_{i}, \mu\right)$ and $0<\beta_{i} \leq 1$. Let $I_{\alpha ; \vec{b}}$ be as in (1.7). Suppose that $0<\alpha+$ $\sum_{i=1}^{m} \beta_{i}<n$ and $1 / q=1-\left(\alpha+\sum_{i=1}^{m} \beta_{i}\right) / n$, then $I_{\alpha ; \vec{b}}$ is bounded from $H^{1}(\mu)$ to $L^{q}(\mu)$ with the operator norm at most $C\left\|b_{1}\right\|_{L i p\left(\beta_{1}\right)} \cdots\left\|b_{m}\right\|_{L i p\left(\beta_{m}\right)}$.

The following results are true only for commutators defined by (1.8) and can not extend to the case $m \geq 2$. 
Theorem 4.3. Let $0<\alpha<n$ and $b \in \operatorname{Lip}(\beta, \mu)$ for $0<\beta \leq 1$. Let $I_{\alpha ; b}$ be as in (1.8). Suppose $n /(\alpha+\beta)<p<n / \alpha$. Then there exists a constant $C>0$ such that for all bounded functions $f$ with compact support,

$$
\left\|I_{\alpha ; b}(f)\right\|_{L i p(\beta+\alpha-n / p)} \leq C\|b\|_{\operatorname{Lip}(\beta)}\|f\|_{L^{p}(\mu)} .
$$

Theorem 4.4. Let $0<\alpha<n$ and $b \in \operatorname{Lip}(\beta, \mu)$ for $0<\beta \leq 1$. Let $I_{\alpha ; b}$ be as in (1.8). Then there exists a constant $C>0$ such that for all bounded functions $f$ with compact support,

$$
\left\|I_{\alpha ; b}(f)\right\|_{R B M O(\mu)} \leq C\|b\|_{L i p(\beta)}\|f\|_{L^{n /(\alpha+\beta)}(\mu)} .
$$

Theorem 4.5. Let $0<\alpha<n$ and $b \in \operatorname{Lip}(\beta, \mu)$ for $0<\beta<1$. Let $I_{\alpha ; b}$ be as in (1.8). Then the following statements are equivalent.

(i) There exists a constant $C>0$ such that for all bounded functions $f$ with compact support,

$$
\left\|I_{\alpha ; b}(f)\right\|_{L i p(\beta)} \leq C\|b\|_{L i p(\beta)}\|f\|_{L^{n / \alpha}(\mu)} .
$$

(ii) There exists a constant $C>0$ such that the function $b$ satisfies the following conditons: For any cube $Q$ and $u \in Q$,

$$
\begin{aligned}
& {\left[\frac{1}{\mu(Q)} \int_{Q}\left|b(x)-m_{Q}(b)\right| d \mu(x)\right]\left|\int_{\mathbb{R}^{d} \backslash 2 Q} K(u, y) f(y) d \mu(y)\right|} \\
& \quad \leq C\|f\|_{L^{n / \alpha}(\mu)} l(Q)^{\beta},
\end{aligned}
$$

and for any cube $R$ such that $Q \subset R$ and $l(R) \leq 2 l(Q)$ and $v \in R$,

$$
\left|m_{R}(b)-m_{Q}(b)\right|\left|\int_{\mathbb{R}^{d} \backslash 2 R} K(v, y) f(y) d \mu(y)\right| \leq C\|f\|_{L^{n / \alpha}(\mu)} l(Q)^{\beta} .
$$

The proofs of Theorem 4.3 and Theorem 4.5 are just linguistic iterations with a slight modification of the proofs of Theorem 2.2 and Theorem 2.4. Moreover, Theorem 4.4 can be deduced from Theorem 4.2 by a standard dual argument. We leave all the details to the reader.

\section{ACKNOWLEDGMENT}

The second (corresponding) author was supported by NNSF (No. 10271015) and RFDP (No. 20020027004) of China. 


\section{REFERENCES}

1. W. Chen and E. T. Sawyer, A note on commutators of fractional integrals with $R B M O(\mu)$ functions, Illinois Math. J. 46 (2002), 1287-1298.

2. J. García-Cuerva and A. E. Gatto, Lipschitz spaces and Calderon-Zygmund operators associated to non-doubling measures, Publ. Mat., 49 (2005), 285-296.

3. J. García-Cuerva and A. E. Gatto, Boundedness properties of fractional integral operators associated to non-doubling measures, Studia Math. 162 (2004), 245-261.

4. J. García-Cuerva and J. Martell, Two-weight norm inequalities for maximal operators and fractional integrals on non-homogeneous spaces, Indiana Univ. Math. J. $\mathbf{5 0}$ (2001), 1241-1280.

5. E. Harboure, C. Segovia and J. L. Torrea, Boundedness of commutators of fractional and singular integrals for the extreme values of p, Illinois J. Math. 41 (1997), 676-700.

6. G. Hu, Y. Meng and D. Yang, Multilinear commutators of singular integrals with non doubling measures, Integral Equations Operator Theory, 51 (2005), 235-255.

7. G. Hu, Y. Meng and D. Yang, New atomic characterization of $H^{1}$ space with nondoubling measures and its applications, Math. Proc. Cambridge Philos. Soc., 138 (2005), 151-171.

8. S. T. Krantz and S. Y. Li, Boundedness and compactness if integral operators on spaces of homogeneous type and applications. I, J. Math. Anal. Appl. 258 (2001), 629-641.

9. S. T. Krantz and S. Y. Li, Boundedness and compactness if integral operators on spaces of homogeneous type and applications. II, J. Math. Anal. Appl. 258 (2001), 642-657.

10. S. Lu, Q. Wu and D. Yang, Boundedness of commutators on Hardy type spaces, Sci. China (Ser. A) 45 (2002), 984-997.

11. F. Nazarov, S. Treil and A. Volberg, Cauchy integral and Calderon-Zygmund operators on nonhomogeneous spaces, Internat. Math. Res. Notices 15 (1997), 703-726.

12. F. Nazarov, S. Treil and A. Volberg, Weak type estimates and Cotlar inequalities for Calderon-Zygmund operators on nonhomogeneous spaces, Internat. Math. Res. Notices 9 (1998), 463-487.

13. F. Nazarov, S. Treil and A. Volberg, Accretive system $T b$-theorems on nonhomogeneous spaces, Duke Math. J. 113 (2002), 259-312.

14. J. Orobitg and C. Pérez, $A_{p}$ weights for nondoubling measures in $\mathbb{R}^{n}$ and applications, Trans. Amer. Math. Soc. 354 (2002), 2013-2033.

15. X. Tolsa, Cotlar's inequality without the doubling condition and existence of principal values for the Cauchy integral of measures, J. Reine Angew. Math. 502 (1998), 199235. 
16. X. Tolsa, A $T(1)$ theorem for non-doubling measures with atoms, Proc. London Math. Soc. 82 (2001), 195-228.

17. X. Tolsa, BMO, $H^{1}$ and Calderon-Zygmund operators for non doubling measures, Math. Ann. 319 (2001), 89-149.

18. X. Tolsa, Littlewood-Paley theory and the $T(1)$ theorem with non-doubling measures, Adv. Math. 164 (2001), 57-116.

19. X. Tolsa, The space $H^{1}$ for nondoubling measures in terms of a grand maximal operator, Trans. Amer. Math. Soc. 355 (2003), 315-348.

20. X. Tolsa, A proof of the weak $(1,1)$ inequality for singular integrals with non doubling measures based on a Calderon-Zygmund decomposition, Publ. Mat. 45 (2001), 163174.

21. X. Tolsa, Painlevé's problem and the semiadditivity of analytic capacity, Acta Math. 190 (2003), 105-149.

22. J. Verdera, The fall of the doubling condition in Calderon-Zygmund theory, Publ. Mat. Vol. Extra (2002), 275-292.

Yan Meng and Dachun Yang

School of Mathematics Sciences,

Beijing Normal University,

Beijing 100875,

People's Republic of China

E-mails: mengyan@mail.bnu.edu.cn

E-mails: dcyang@bnu.edu.cn 